\title{
Open-Label Allopregnanolone Treatment of Men with Fragile X-Associated Tremor/Ataxia Syndrome
}

\author{
J. Y. Wang ${ }^{1,2}$ • A. M. Trivedi ${ }^{3}$ N. R. Carrillo ${ }^{3}$ J. Yang ${ }^{4,5}$ - A. Schneider ${ }^{1,6}$. \\ C. Giulivi ${ }^{1,7} \cdot$ P. Adams ${ }^{1,6} \cdot$ F. Tassone ${ }^{1,2} \cdot$ K. Kim $^{1,8} \cdot$ S. M. Rivera ${ }^{1,5,9} \cdot$ N. Lubarr $^{10}$ • C.- \\ Y. Wu ${ }^{4,11} \cdot$ R. W. Irwin ${ }^{12} \cdot$ R. D. Brinton ${ }^{12,13}$ - J. M. Olichney ${ }^{4,5}$ - M. A. Rogawski ${ }^{4,14}$. \\ R. J. Hagerman ${ }^{1,6}$
}

Published online: 13 July 2017

(C) The American Society for Experimental NeuroTherapeutics, Inc. 2017

\begin{abstract}
Fragile X-associated tremor/ataxia syndrome (FXTAS) is a late-onset neurodegenerative disorder affecting approximately $45 \%$ of male and $16 \%$ of female carriers of the FMR1 premutation over the age of 50 years. Currently, no effective treatment is available. We performed an open-label intervention study to assess whether allopregnanolone, a neurosteroid promoting regeneration and repair, can improve clinical symptoms, brain activity, and magnetic resonance imaging (MRI) measurements in patients with FXTAS. Six patients underwent weekly intravenous infusions of allopregnanolone (2-6 mg over $30 \mathrm{~min}$ ) for 12 weeks. All patients completed baseline and follow-up studies, though MRI scans were not collected from 1 patient because of MRI contraindications. The MRI scans from previous visits, along
\end{abstract}

R. J. Hagerman

rjhagerman@ucdavis.edu

1 UC Davis MIND Institute, UC Davis Health, Sacramento, CA, USA

2 Department of Biochemistry and Molecular Medicine, School of Medicine, University of California, Davis, Sacramento, CA, USA

3 School of Medicine, University of California, Davis, Sacramento, CA, USA

4 Department of Neurology, School of Medicine, University of California, Davis, Sacramento, CA, USA

5 Center for Mind and Brain, University of California, Davis, Davis, CA, USA

6 Department of Pediatrics, School of Medicine, University of California, Davis, Sacramento, CA, USA

7 Department of Molecular Biosciences, University of California Davis, School of Veterinary Medicine, Davis, CA, USA with scans from 8 age-matched male controls, were also included to establish patients' baseline condition as a reference. Functional outcomes included quantitative measurements of tremor and ataxia and neuropsychological evaluations. Brain activity consisted of event-related potential N400 word repetition effect during a semantic memory processing task. Structural MRI outcomes comprised volumes of the hippocampus, amygdala, and fluid-attenuated inversion recovery hyperintensities, and microstructural integrity of the corpus callosum. The results of the study showed that allopregnanolone infusions were well tolerated in all subjects. Before treatment, the patients disclosed impairment in executive function, verbal fluency and learning, and progressive deterioration of all MRI measurements. After treatment,
8 Department of Public Health Sciences, University of California, Davis, Davis, CA, USA

9 Department of Psychology, University of California Davis, Davis, CA, USA

10 Department of Neurology, Mount Sinai Beth Israel Hospital, New York, NY, USA

11 PK/PD Bioanalytical Core Facility, UC Davis Health, Sacramento, CA, USA

12 Department of Pharmacology and Pharmaceutical Sciences, School of Pharmacy, University of Southern California, Los Angeles, CA, USA

13 Center for Innovation in Brain Science, School of Medicine, Departments of Pharmacology and Neurology, University of Arizona, Tucson, AZ, USA

14 Department of Pharmacology, School of Medicine, University of California, Davis, Sacramento, CA, USA 
the patients demonstrated improvement in executive functioning, episodic memory and learning, and increased N400 repetition effect amplitude. Although MRI changes were not significant as a group, both improved and deteriorated MRI measurements occurred in individual patients in contrast to uniform deterioration before the treatment. Significant correlations between baseline MRI measurements and changes in neuropsychological test scores indicated the effects of allopregnanolone on improving executive function, learning, and memory for patients with relatively preserved hippocampus and corpus callosum, while reducing psychological symptoms for patients with small hippocampi and amygdalae. The findings show the promise of allopregnanolone in improving cognitive functioning in patients with FXTAS and in partially alleviating some aspects of neurodegeneration. Further studies are needed to verify the efficacy of allopregnanolone for treating FXTAS.

Keywords Fragile X premutation $\cdot F M R 1$.

Neurodegeneration $\cdot$ Allopregnanolone $\cdot$ FXTAS ·

Neurogenesis

\section{Introduction}

Fragile X-associated tremor/ataxia syndrome (FXTAS) is a late-adult-onset neurodegenerative disorder that affects, with age- and sex-specific penetrance, carriers of premutation alleles (55-200 CGG repeats) of the fragile X mental retardation 1 (FMR1) gene. Defining clinical features of FXTAS include progressive kinetic tremor, gait ataxia, executive function and memory deficits, peripheral neuropathy, and parkinsonism. Associated variable features include cognitive impairment and dementia; psychiatric symptoms, such as depression; and dysautonomia [1-4]. FXTAS is characterized by progressive global brain atrophy and white matter disease of both the cerebellum and cerebrum [5-8]. Cerebellar and brainstem atrophy and ventricular enlargement are detectable even before the onset of tremor or ataxia $[6,9,10]$. Currently, no specific treatment for FXTAS can slow the progression of neurodegeneration, though symptomatic treatments exist for some of the symptoms, such as tremor [11].

Allopregnanolone is a naturally occurring neurosteroid with activity as a positive modulator of $\gamma$-aminobutryic acid A receptors that can stimulate hippocampal neurogenesis and reverse hippocampal-dependent learning and memory dysfunction [12]. Allopregnanolone also promotes oligodendrogenesis and myelination [13] and confers neuroprotection by reducing the expression of the proapoptotic protein caspase $3[14,15]$. We recently reported that hippocampal neurons cultured from premutation mice exhibit abnormal network bursting [16]. Such persistent bursting is a plausible mechanism for the neurodegeneration that leads to the neurological symptoms in FXTAS. We have further demonstrated that allopregnanolone, by potentiating $\gamma$-aminobutryic acid Areceptor signaling, eliminates the abnormal bursting in the premutation neurons, providing a mechanism for neuroprotection [16].

Allopregnanolone is endogenously present in the periphery and brain, and thus is expected to be safe for long-term treatment. Intravenous infusion of allopregnanolone in healthy subjects [17] and in the treatment of status epilepticus [18, 19] has not revealed treatment-related adverse effects other than sedation and mild nausea. Given the neuroregenerative properties of allopregnanolone and the preclinical evidence of efficacy in the FXTAS model, we sought to conduct a preliminary, open-label investigation of the therapeutic potential of allopregnanolone for carriers of the premutation with FXTAS. We assessed the impact of allopregnanolone on working memory deficits and neurological symptoms. In addition, we explored whether improvements in mitochondrial function and magnetic resonance imaging (MRI) measurements can be observed that reflect neuroregeneration and alleviation of white matter disease.

\section{Methods}

\section{Study Design and Patients}

This study was a preliminary, 3-month, open-label, uncontrolled trial of allopregnanolone treatment in 6 men with FXTAS who received weekly intravenous infusions for a period of 12 weeks. Patients with FXTAS were recruited at the University of California, Davis (UC Davis), MIND Institute's Fragile X Research and Treatment Center between December 2015 and March 2016, with the last participant completing treatment in July 2016. Notification of the study was provided on ClinicalTrials.gov (Identifier: NCT02603926).

Individuals aged 50-85 years with the premutation and a diagnosis of FXTAS were eligible for the study. Exclusion criteria were other serious systemic disease, alcohol or drug abuse, and current use of phenytoin. To establish patients' baseline condition, we included scans acquired before the treatment. Table 1 summarizes demographic information for the enrollees. Serial brain MRI scans from 8 age-matched healthy men carrying normal FMR1 alleles (CGG repeat length 20-32) were used as a null reference group for assessing MRI parameters (age $61 \pm 6$ years, range $52-72$ years, 2-3 scans/individual).

CGG-repeat sizing was carried out using a polymerase chain reaction-based approach [20]. Mitochondrial outcomes in lymphocytes were measured, as previously described [21]. Lactate and pyruvate ratios from lymphocytes were detected by utilizing a metabolomic approach. 
Table 1 Demographic information

\begin{tabular}{lllllll}
\hline Participants & Age & $\begin{array}{l}\text { Total no. of previous } \\
\text { visits }\end{array}$ & $\begin{array}{l}\text { CGG-repeat } \\
\text { length }\end{array}$ & $\begin{array}{l}\text { FXTAS } \\
\text { stage }\end{array}$ & $\begin{array}{l}\text { Education } \\
\text { (years) }\end{array}$ & Handedness \\
\hline Patient 1 & 57 & 1 & 104 & 5 & 16 & Right \\
Patient 2 & 68 & 3 & 88 & 4 & 27 & Right \\
Patient 3 & 79 & 0 & 83 & 3 & 20 & Right \\
Patient 4 & 64 & 2 & 105 & 4 & 20 & Right \\
Patient 5 & 68 & 2 & 85 & 3 & 17 & Right \\
Patient 6 & 74 & 2 & 98 & 5 & 20 & Right \\
\hline
\end{tabular}

FXTAS $=$ Fragile $\mathrm{X}$-associated tremor/ataxia syndrome

\section{Intervention}

The study was conducted under US Food and Drug Administration-approved Investigational New Drug Applications 111,085 (to MAR) and 125,502 (to RJH) and with the consent of the UC Davis Institutional Review Board. Written informed consent was also obtained from each patient prior to the study. Allopregnanolone intravenous injection was formulated at the UC Davis Good Manufacturing Practices Laboratory and dispensed by the UC Davis Investigational Drug Pharmacy. The formulation consisted of $99.9 \%$ pure GMP-grade allopregnanolone ( $3 \alpha$-hydroxy$5 \alpha$-pregnan-20-one) at a concentration of $0.5 \mathrm{mg} / \mathrm{ml}$ in $0.9 \%$ sodium chloride injection, United States Pharmacopeia, containing $6 \%$ sulfobutyl ether- $\beta$-cyclodextrin sodium salt, United States Pharmacopeia (Dexolve; CycloLab, Budapest, Hungary). The weekly intravenous infusions and follow-up observations were performed at the Infusion Center at the UC Davis CTSC Clinical Research Center (CCRC) except for 2 patients residing in the New York area who received their first 3 infusions at the CCRC Infusion Center and the remainder of their infusions at the Mount Sinai Beth Israel Hospital Therapeutic Infusion Center (New York, NY, USA) under the supervision of one of the co-authors of this study (NL).

All infusions were administered over $30 \mathrm{~min}$, followed by a flush for $30 \mathrm{~min}$. The dose for the first infusion was $2.0 \mathrm{mg}$; the subsequent week, the dose was increased to $4.0 \mathrm{mg}$; and finally increased to $6.0 \mathrm{mg}$ in the third week. The Richmond Agitation Sedation Scale (RASS) was administered before, during, and after each infusion. Infusions were to be terminated if the score fell below -1 (drowsy) during the infusion. Each subject had a RASS score of 0 (alert and calm) before beginning infusion and maintained the RASS score of 0 throughout each infusion. Since the 6.0-mg dose was tolerated by all subjects, this dose was administered on weeks 3 to 12 in all cases.

Each participant was required to be accompanied by a chaperone, who drove the participant home from the CCRC Infusion Center and remained with the participant for at least $20 \mathrm{~h}$, to monitor for adverse effects.

\section{Blood Level Measurements and Pharmacokinetic Analysis}

Plasma allopregnanolone levels were determined by liquid chromatography-tandem mass spectrometry in 1 subject in blood samples drawn at intervals during and after a 6-mg infusion from the opposite arm of that receiving the intravenous infusion. Blood was collected in heparinized tubes before infusion (0 time), at 15 and $30 \mathrm{~min}$ during the infusion, and at 30, 90, and $150 \mathrm{~min}$ after the infusion. The blood was centrifuged at $1500 \mathrm{~g}$ for $10 \mathrm{~min}$ to separate the plasma, which was stored at $-80{ }^{\circ} \mathrm{C}$ until the time of analysis. D4allopregnanolone was added as internal standard, and the analytes were extracted using the solid phase extraction method and injected into a Waters ACQUITY UPLC System (Waters, Milford, MA, USA). The effluent was directed to a Xevo TQ-S triple quadrupole mass spectrometer, which was used to ionize target molecules and monitor the ion $\mathrm{m} / \mathrm{z}$ transitions from $319.20 \rightarrow 283.30$ for allopregnanolone quantification and $323.3 \rightarrow 287.3$ for D4-allopregnanolone quantification. Two-compartment pharmacokinetic parameters were estimated with Phoenix WinNonlin 7.0 (Certara, Princeton, NJ, USA).

\section{Assessments and Follow-Up}

\section{Clinical Assessment}

All participants completed baseline and follow-up clinical examinations and neuropsychological assessments. A detailed physical and neurological examination was performed and videotaped [22], emphasizing the major features of FXTAS: cerebellar ataxia, intention tremor, and neuropathy findings, such as vibration sense and pinprick sensation.

\section{Neuropsychological and Emotional Assessment}

Learning and memory was measured through the California Verbal Learning Test 2 [23]. Working memory was assessed using Weschler Memory Scale-IV (WMS-IV) [24]. Other cognitive measures included Mini Mental Status Exam 
(MMSE) [25], Behavior Dyscontrol Scale (BDS-2) [26, 27], Cambridge Neuropsychological Test Automated Battery (CANTAB) [28], and Controlled Oral Word Association Test [29]. The Symptom Checklist-90-Revised (SCL-90-R) [30], and Beck Anxiety Inventory [31] were used to assess neuropsychological symptoms. In addition, CATSYS $[32,33]$ tremor and sway assessments evaluated improvements in hand tremor and balance. Table 2 shows a brief description of functional measures used in the study.

\section{MRI Acquisitions and Analyses}

MRI scans were acquired on a Siemens Trio 3 T MRI scanner (Siemens Medical Solutions, Erlangen, Germany) equipped with a 32-channel head coil. High-resolution T1-weighted Magnetization Prepared Rapid Gradient Echo (MPRAGE) images were acquired in 192 sagittal slices of 1-mm thickness with field of view (FOV) $256 \mathrm{~mm}, 256 \times 256$ matrix, repetition time (TR) of $2170 \mathrm{~ms}$, echo time (TE) of $4.82 \mathrm{~ms}$, and $7^{\circ}$ flip angle. Fluid attenuated inversion recovery (FLAIR) images for quantifying subcortical lesions were acquired in 104 sagittal slices of 1.9-mm thickness with FOV $243 \mathrm{~mm}, 512 \times$ 512 matrix, TR of $5000 \mathrm{~ms}$, TE of $456 \mathrm{~ms}$, and inversion time $1700 \mathrm{~ms}$. Diffusion tensor imaging (DTI) scans for assessing microstructural integrity were obtained using a single shot spin-echo echo planar imaging (EPI) sequence in 68 axial slices of 2-mm thickness with FOV $224 \mathrm{~mm}, 112 \times 112$ matrix zero-filled to $224 \times 224$ pixels, TR of $7100 \mathrm{~ms}$, TE of $73 \mathrm{~ms}$, number of excitations of 2, 30 gradient encoding directions for $b=800 \mathrm{~s} / \mathrm{mm}^{2}$, along with 4 interleaved $b=0 \mathrm{~s} / \mathrm{mm}^{2}$ images.

MRI preprocessing included automated anterior commissure-posterior commissure alignment in T1-weighted scans using acpcdetect (http://www.nitrc.org/projects/art) [34]. For failed cases, manual anterior commissure-posterior commissure alignment was performed using DTI Studio (www.mristudio.org) [35]. MRI bias field correction in both $\mathrm{T} 1$ and FLAIR scans were performed using N4 (http://stnava. github.io/ANTs/) [36]. For DTI scans, motion and eddy current were corrected using FSL (http://fsl.fmrib.ox.ac.uk/fsl/fslwiki/) [37] prior to the generation of fractional anisotropy (FA) and mean diffusivity (MD) maps using DTI Studio.

Hippocampus, amygdala, and corpus callosum were segmented automatically using MRI Cloud (http://mricloud.org) [38], followed by automated machine learning-based error correction implemented in SegAdapter (https://www.nitrc.org/ projects/segadapter/) $[39,40]$ and manual corrections. DTI FA and MD values of the corpus callosum were calculated based on previously published method [41], with modifications. Within-subject co-registration of MD maps with $\mathrm{T} 1$ was performed automatically, and then manually adjusted to maximize the match of the corpus callosum using FreeSurfer (https:// surfer.nmr.mgh.harvard.edu/fswiki/bbregister) [42]. The resulting transformation was then applied to the FA maps. To further reduce the effect of mismatch, we generated masks by thresholding the MD maps at $2 \mu \mathrm{m}^{2} / \mathrm{ms}$ and applied the masks to both FA and MD maps using the FSL command, fslmaths. The generation of regions-of-interests for the 3 brain structures (i.e., hippocampus, amygdala, and corpus callosum), and calculation of volume, FA, and MD values were also performed in FSL using the commands fslmaths and fslstats.

Segmentation of subcortical hyperintensities was performed on FLAIR scans using a fully automated tool, lesion prediction algorithm (LPA) (http://www.applied-statistics.de/ 1st.html) [43] from SPM12. LPA has shown high performance relative to other publically available tools for automated FLAIR lesion segmentation [44]. To calculate FLAIR hyperintensity volume (HV) in the corpus callosum, we applied the threshold of 0.7 to LPA-generated lesion probability

Table 2 Description of neuropsychological and emotional tests

Outcome measures

Beck Anxiety Inventory (BAI)

Behavioral Dyscontrol Scale (BDS-2)

Cambridge Neuropsychological Test Automated Battery (CANTAB) CATSYS

Controlled Oral Word Association Test (COWAT)

California Verbal Learning Test (CVLT-II)

Mini Mental Status Exam (MMSE)

Symptom Checklist-90-Revised (SCL-90-R)

Weschler Memory Scale Fourth Edition (WMS-IV)
Description

Self-report questionnaire to obtain measurement of anxiety

Nine-item measurements assessing frontal lobe integrity, including executive function and cognitive control over behaviors

Computerized test measuring memory, attention and executive function

Test measuring postural sway, tremor, and gait using force plate and tremor pen

Assessment measuring verbal fluency or cognitive flexibility that can be correlated with executive functioning

Assessment measuring working memory and memory subtypes

Clinician-administered assessment measuring cognitive function, mental state, orientation, and memory

Ninety-item self-reporting measure of psychological symptoms

Assessment measuring immediate, delayed, visual and auditory memory 
maps, followed by applying corpus callosum masks using fslmaths.

\section{Event-Related Potential Word Repetition Effect Assessment}

Electroencephalogram (EEG) recordings were obtained during a semantic category decision/word repetition paradigm at both visits [45]. Categorical statements were read aloud by an experimenter, with each phrase followed ( $\sim \mathrm{s}$ later) by a target word visually presented in the center of the monitor (duration $=$ $300 \mathrm{~ms}$, visual angle $=0.4^{\circ}$ ). Participants were instructed to not move or respond for $3 \mathrm{~s}$ following the target word. Their response was to repeat the spoken word, followed by a "yes" or "no" judgment indicating whether the word was congruent with the preceding statement (congruous trial, 50\%) or not (incongruous trial, 50\%). An EEG session consisted of 2 blocks of 144 trials, each block lasting approximately $20 \mathrm{~min}$. In each block, 12 congruous category-target pairs were presented once, 12 presented twice, and 12 presented 3 times. Equal numbers of incongruous pairs were presented in the same repetition conditions.

Thirty-two-channel EEG was recorded (Nicolet SM 2000 amplifier, bandpass $0.016-100 \mathrm{~Hz}$, sampling rate $250 \mathrm{~Hz}$ ). Interelectrode impedance was maintained below $5 \mathrm{k} \Omega$. All scalp electrodes were referenced online to the left mastoid and rereferenced offline to the average of both mastoids. Eye movements were captured by electro-oculogram recorded with 4 electrodes ( 1 beneath and 1 at the outer canthus of each eye). All trials free of EEG/electro-oculogram artifact were averaged to produce the event-related potential (ERP) for each participant session.

\section{Statistical Analysis}

All statistical analyses were conducted in R 3.2.5 language and environment [46]. To establish baseline MRI measurements for patients with FXTAS, group means and change rates before treatment were compared with the healthy controls using mixed effects models [47]. Improvements in primary outcome measures before and after treatment were tested for significance using 1sided paired $t$ tests. Pearson correlation coefficients were utilized to identify pretreatment MRI data predictive of functional improvements after the treatment. The threshold for statistical significance was set at 0.05 .

\section{Results}

Patient 3 (body weight $76.3 \mathrm{~kg}$ ) received an intravenous infusion of $6 \mathrm{mg}$ allopregnanolone over $30 \mathrm{~min}$. Blood was collected in heparinized tubes before infusion ( 0 time), at 15 and 30 min during the infusion, and at 30,90, and 150 min after the infusion. Table 3 shows the estimated 2-compartment pharmacokinetic parameters. The peak allopregnanolone
Table 3 Estimated 2compartment pharmacokinetic parameters

\begin{tabular}{lll} 
Parameter & Estimate & Units \\
\hline $\mathrm{V}$ & 1.11 & $1 / \mathrm{kg}$ \\
$\mathrm{V} 2$ & 1.61 & $1 / \mathrm{kg}$ \\
$\mathrm{CLx}$ & 2.12 & $1 / \mathrm{h} / \mathrm{kg}$ \\
$\mathrm{CL} 2$ & 1.87 & $1 / \mathrm{h} / \mathrm{kg}$ \\
\hline
\end{tabular}

$\mathrm{T}_{\max }=30 \mathrm{~min} . \mathrm{C}_{\max }=35.5 \mathrm{ng} / \mathrm{ml}$. The falling phase was fit to a single exponential with half-time of $34.7 \mathrm{~min}$

$\mathrm{V}=$ volume of distribution of central compartment; V2 = volume of distribution of peripheral compartment; $\mathrm{CL}=$ central compartment clearance; CL2 = peripheral compartment clearance

concentration achieved at the end of the 30-min infusion was $35.5 \mathrm{ng} / \mathrm{ml}(112 \mathrm{nM})$. The plasma concentration values are shown in Figure 1.

\section{Functional Outcomes}

At baseline, all patients exhibited impairment in executive function assessed using BDS-2 (range 8-17). Four patients showed impairment in learning and memory (California Verbal Learning Test-II T-score 24-37) and 4 (with 3 overlapping) disclosed compromised verbal fluency (Controlled Oral Word Association Test-total raw score 16-29). In addition, 2 patients showed borderline scores of WMS visual memory (73 and 79) and 1 with borderline MMSE score at 24.

Table 4 shows the comparisons of functional outcome measures before and after the treatment. The majority of the patients showed improvement in mental state (MMSE), executive function (BDS-2 and CANTAB One Touch Stockings), memory (WMS-IV and CANTAB Paired Associates Learning), anxiety (SCL-90-R), and intention tremor (CATSYS Dot-to-Dot left-hand tremor intensity); improvement in BDS-2 and CANTAB Paired Associates Learning total error adjusted achieved statistical significance $(p=$ 0.009 and $p=0.042$, respectively).

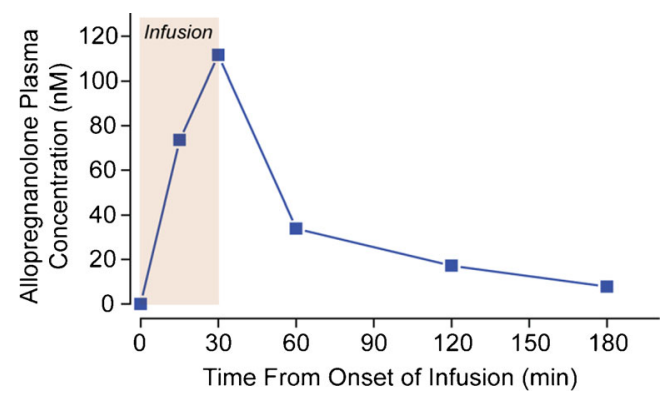

Fig. 1 Allopregnanolone plasma concentrations and pharmacokinetic analysis in patient 3 
Table 4 Outcome measurements before and after treatment

\begin{tabular}{|c|c|c|c|c|c|c|}
\hline \multirow[t]{2}{*}{ Outcome measurements } & \multirow[t]{2}{*}{$n$} & \multirow{2}{*}{$\begin{array}{l}\text { Before } \\
\text { infusion } \\
\text { Mean } \pm \text { SD }\end{array}$} & \multirow{2}{*}{$\begin{array}{l}\text { After } \\
\text { infusion } \\
\text { Mean } \pm \text { SD }\end{array}$} & \multirow[t]{2}{*}{$\begin{array}{l}\% \text { of patients } \\
\text { improved }\end{array}$} & \multicolumn{2}{|c|}{$\begin{array}{l}\text { Paired } t \text { test } \\
\text { (1-sided) }\end{array}$} \\
\hline & & & & & $t$ & $p$-value \\
\hline MMSE & 6 & $27.3 \pm 2.3$ & $28.7 \pm 0.8$ & 67 & 1.87 & 0.06 \\
\hline BDS-2 & 6 & $12.7 \pm 3.1$ & $15.3 \pm 3.8$ & 100 & 3.51 & 0.009 \\
\hline WMS-IV Auditory Memory & 6 & $92.5 \pm 8.4$ & $94.2 \pm 11.2$ & 50 & 0.62 & 0.28 \\
\hline WMS-IV Visual Memory & 5 & $83.2 \pm 9.3$ & $89.6 \pm 14.4$ & 80 & 1.31 & 0.13 \\
\hline WMS-IV Immediate Memory & 5 & $88.6 \pm 8.6$ & $93.0 \pm 11.3$ & 80 & 1.43 & 0.11 \\
\hline WMS-IV Delayed Memory & 5 & $88.2 \pm 8.4$ & $91.2 \pm 8.5$ & 80 & -0.67 & 0.73 \\
\hline CVLT-II & 6 & $34.0 \pm 7.5$ & $33.5 \pm 6.3$ & 33 & -0.28 & 0.61 \\
\hline COWAT & 6 & $26.2 \pm 6.7$ & $26.2 \pm 4.0$ & 33 & 0 & 0.50 \\
\hline Beck Anxiety Inventory & 4 & $7.0 \pm 7.4$ & $4.5 \pm 4.1$ & 50 & -1.35 & 0.14 \\
\hline SCL-90 Global Severity Index & 4 & $55.8 \pm 14.5$ & $51.3 \pm 7.8$ & 75 & -0.78 & 0.25 \\
\hline CANTAB OTS PSFC & 5 & $6.60 \pm 1.52$ & $7.17 \pm 2.14$ & 60 & 2.06 & 0.054 \\
\hline CANTAB OTS MLFC (s) & 5 & $52 \pm 24.9$ & $55.9 \pm 41.9$ & 60 & 0.42 & 0.65 \\
\hline CANTAB PAL Total Errors (Adj.) & 6 & $76.5 \pm 44.2$ & $58.2 \pm 38.8$ & 83 & -2.15 & 0.042 \\
\hline CANTAB SWM Total Errors & 6 & $63.8 \pm 17.2$ & $66.5 \pm 6.7$ & 50 & 0.31 & 0.62 \\
\hline CATSYS DTD TI, right hand (m/s) & 6 & $0.87 \pm 0.49$ & $0.79 \pm 0.59$ & 50 & -1.08 & 0.16 \\
\hline CATSYS DTD TI, left hand (m/s) & 6 & $0.74 \pm 0.29$ & $0.65 \pm 0.26$ & 67 & -1.08 & 0.17 \\
\hline CATSYS PS EO, $30 \mathrm{sec}\left(\mathrm{mm}^{2}\right)$ & 4 & $10.1 \pm 0.81$ & $16.0 \pm 13.6$ & 50 & 1.00 & 0.81 \\
\hline Hippocampus $\left(\mathrm{cm}^{3}\right)$ & 5 & $5.87 \pm 1.00$ & $5.84 \pm 1.18$ & 40 & -0.28 & 0.60 \\
\hline Amygdala $\left(\mathrm{cm}^{3}\right)$ & 5 & $3.13 \pm 0.35$ & $3.14 \pm 0.36$ & 40 & 0.17 & 0.44 \\
\hline Subcortical HV $\left(\mathrm{cm}^{3}\right)$ & 5 & $48.9 \pm 29.2$ & $52.8 \pm 24.5$ & 40 & 0.79 & 0.76 \\
\hline Corpus callosum $\left(\mathrm{cm}^{3}\right)$ & 5 & $22.1 \pm 2.6$ & $21.2 \pm 3.6$ & 20 & -1.77 & 0.93 \\
\hline Corpus callosum HV $\left(\mathrm{cm}^{3}\right)$ & 5 & $5.9 \pm 2.6$ & $5.6 \pm 2.3$ & 40 & -0.48 & 0.33 \\
\hline Corpus callosum FA & 5 & $0.53 \pm 0.05$ & $0.54 \pm 0.05$ & 60 & 0.94 & 0.20 \\
\hline Corpus callosum MD $\left(\mu \mathrm{m}^{2} / \mathrm{ms}\right)$ & 5 & $1.03 \pm 0.12$ & $1.04 \pm 0.09$ & 60 & 0.21 & 0.58 \\
\hline N4 Repetition $(\mu \mathrm{V})$ & 4 & $0.38 \pm 0.95$ & $-1.63 \pm 0.14$ & 100 & -4.03 & 0.014 \\
\hline
\end{tabular}

Magnetic resonance imaging measurements before infusion included only the last visits before infusion. Bold values indicated significance (i.e., $p<0.05$ )

MMSE = Mini Mental Status Exam; BDS-2 = Behavioral Dyscontrol Scale; WMS-IV = Weschler Memory Scale Fourth Edition; CVLT-II = California Verbal Learning Test; COWAT $=$ Controlled Oral Word Association Test; SCL-90-R = Symptom Checklist-90-Revised; CANTAB = Cambridge Neuropsychological Test Automated Battery; CANTAB OTS $=$ CANTAB One Touch Stockings; CANTAB OTS PSFC $=$ CANTAB OTS problem solved on first choice; CANTAB OTS MLFC $=$ CANTAB OTS mean latency to first choice; CANTAB PAL = CANTAB Paired Association Learning; CANTAB SWM = CANTAB Spatial Working Memory; CATSYS DTD $\mathrm{TI}=$ CATSYS Dot-to-Dot Tremor Intensity; CATSYS PS EO = CATSYS Postural Sway Eyes Open; HV = hyperintensity volume; $\mathrm{FA}=$ fractional anisotropy; $\mathrm{MD}=$ mean diffusivity

\section{MRI Findings}

MRI analyses were performed on 5 patients from whom both pre- and post-treatment MRI scans were available. Before treatment, the patients showed significant progressive atrophy in the hippocampus, amygdala, and corpus callosum; HV expansion in both subcortical and corpus callosum regions; and FA decline and MD elevation in the corpus callosum ( $p$ from $<0.001$ to 0.045). Compared with the controls, the patients exhibited significantly elevated corpus callosum HV and MD, as well as accelerations in hippocampal atrophy, subcortical HV expansion, and corpus callosum FA decline (Fig. 2). After the treatment,
MRI changes in both directions were seen in individual patients (Fig. 3). However, as a group, none of the changes reached statistical significance at the 0.05 level (Table 4).

\section{Prediction of Cognitive Improvement}

We evaluated whether baseline MRI measurements could predict cognitive improvement after the treatment. Whereas high baseline hippocampal volume was correlated significantly with improvement in both BDS-2 and WMS-IV visual memory $(r=$ 0.89 and $r=0.96$, respectively; $R^{2}=0.79$ and $R^{2}=0.93$, respectively; $p=0.043$ and $p=0.008$, respectively), both low baseline 
Fig. 2 Spaghetti plots showing longitudinal changes in magnetic resonance imaging measurements for premutation carriers before treatment and controls. Orange denotes patients with Fragile Xassociated tremor/ataxia syndrome; blue indicates controls. $\mathrm{HV}=$ hyperintensity volume; $\mathrm{CC}=$ corpus callosum; $\mathrm{FA}=$ frac tional anisotropy; $\mathrm{MD}=$ mean diffusivity
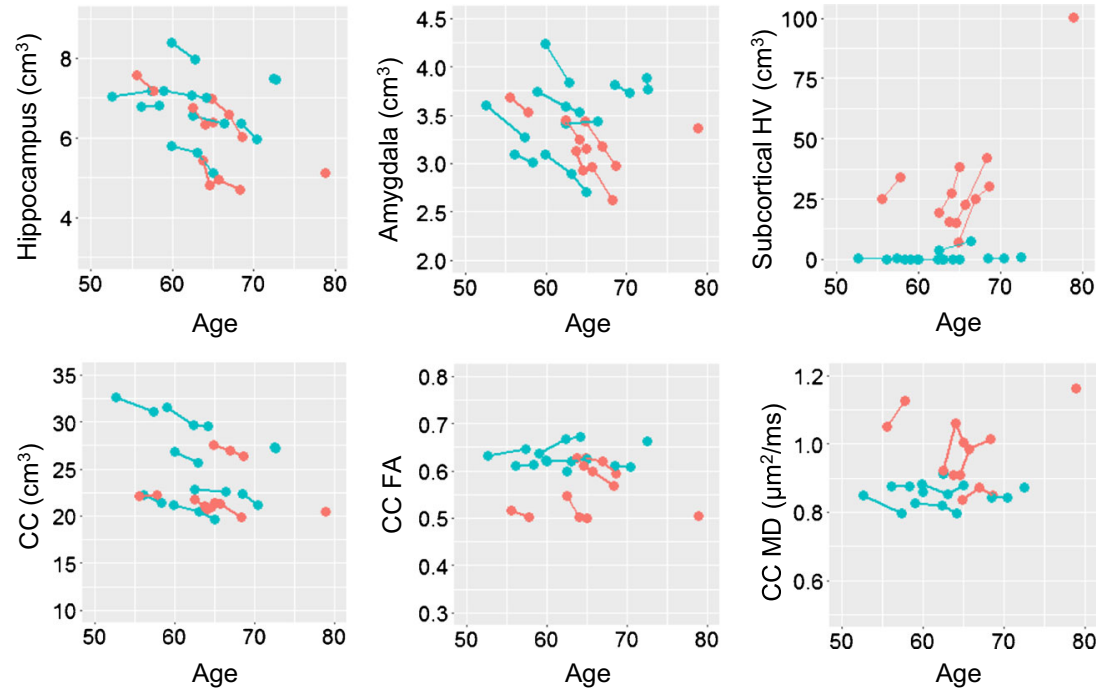

hippocampal and amygdala volumes were correlated significantly with reduced SCL-90-R symptom severity $(r=0.98$ and $r=$ 0.95 , respectively; $R^{2}=0.95$ and $R^{2}=0.91$, respectively; $p=$ 0.023 and $p=0.046$, respectively). In addition, low baseline subcortical HV volume was correlated with improvement in WMSIV immediate memory $\left(r=-0.94, R^{2}=0.88, p=0.018\right)$, and both low baseline corpus callosum HV fraction (i.e., HV/volume) and MD values showed correlations with reduction in CANTAB One Touch Stockings mean latency to first choice ( $r=1.00$ and $r=0.99$, respectively; $R^{2}=1.00$ and $R^{2}=0.97$, respectively; $p<0.001$ and $p=0.013$, respectively) (Fig. 4).

\section{ERP Results}

Figure 5 shows the grand averaged ERP difference waveforms (Incongruous New minus Incongruous Old) for the baseline and follow-up based on 4 patients. At the posterior electrodes illustrated, ERPs at the follow-up visits were more negative in general than those at the baseline. Paired $t$ tests demonstrated that mean amplitudes of the $\mathrm{N} 400$ repetition effect at $\mathrm{POz}$ were larger (more negative) at the follow-up compared with the baseline ( $p=0.014$; Table 4), suggesting beneficial intervention effects on verbal learning and memory.

\section{Molecular Measurements}

None of the mitochondrial outcomes tested (Complex I, II-III, IV, V, and citrate synthase activities; ratios of lactate-to-pyruvate, lactate, pyruvate, ratio of alanine and lactate to pyruvate; shortchain organic acids levels associated with mitochondrial respiratory chain disorders) were significantly different before and after the treatment (as determined by paired $t$ test).

\section{Discussion}

This open-label study demonstrates that intravenous infusion of allopregnanolone at doses up to $6 \mathrm{mg}$ are well tolerated and
Fig. 3 Changes in magnetic resonance imaging measurements before and after allopregnanolone treatment. $\mathrm{HV}=$ hyperintensity volume; $\mathrm{CC}=$ corpus callosum; $\mathrm{FA}=$ fractional anisotropy; $\mathrm{MD}=$ mean diffusivity
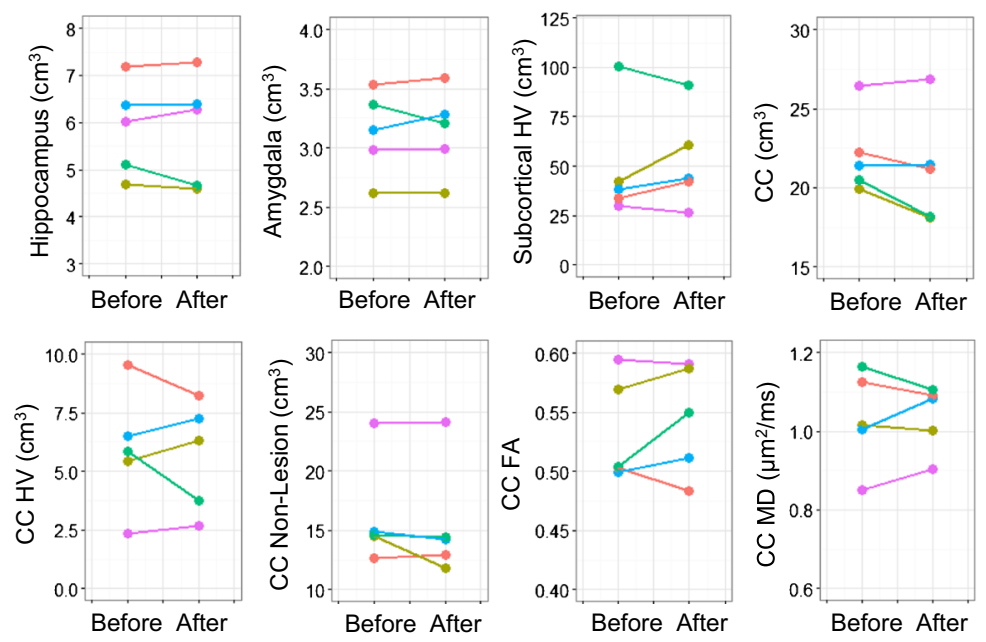
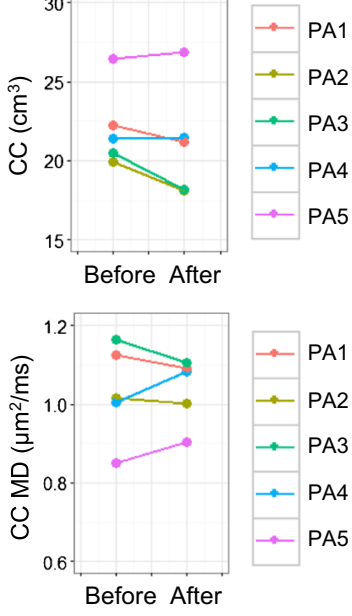

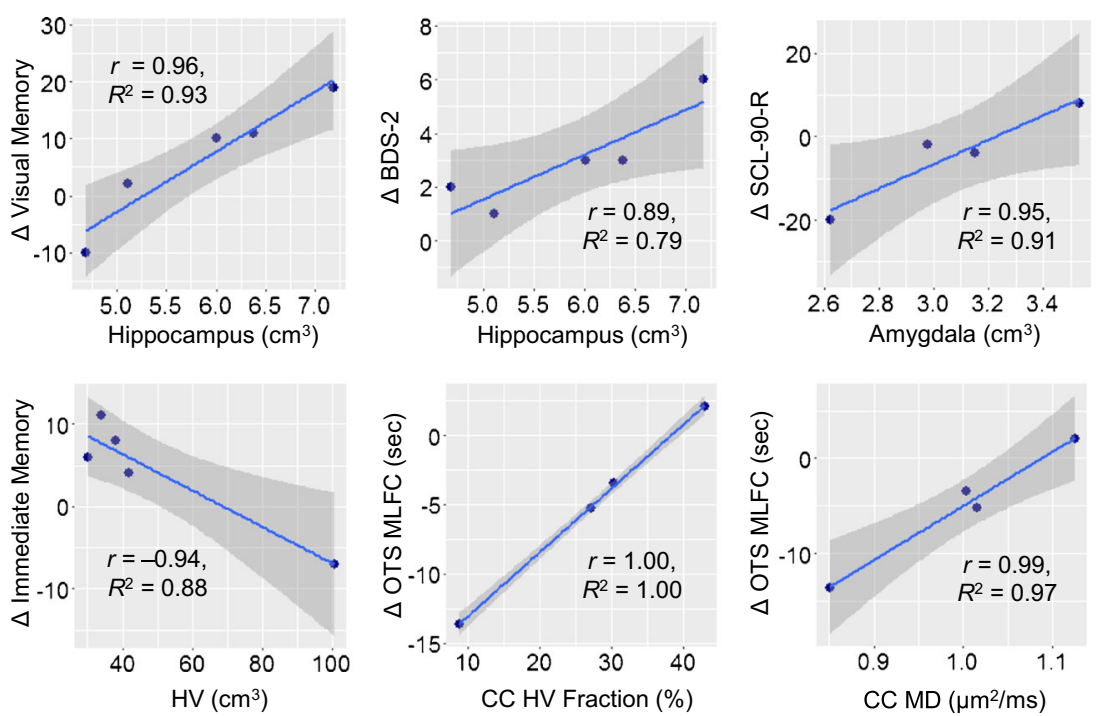

Fig. 4 Correlations between baseline magnetic resonance imaging measurements and changes in cognitive outcomes before and after allopregnanolone treatment. Both correlation of coefficient $(r)$ and coefficient of determination of goodness of fit $\left(R^{2}\right)$ are provided. Shaded areas represent the $95 \%$ confidence intervals on the linear

without apparent adverse effects in men with FXTAS. In a previous study of healthy women, a similar dose of allopregnanolone was associated with sedation and, in some subjects, mild nausea [17]; changes in saccadic eye movements were also consistent with sedation. While the peak concentration of allopregnanolone in patient 3 was modestly higher than achieved in this prior study, our subject did not exhibit sedation or nausea. The half-life of allopregnanolone following termination of the infusion was approximately $35 \mathrm{~min}$. Therefore, the peak level was only present for a brief period of time, thus likely limiting the occurrence of side effects. In contrast, in the prior study, subjects were dosed with regression lines. BDS-2 $=$ Behavioral Dyscontrol Scale; SCL-90-R = Symptom Checklist-90-Revised; HV = hyperintensity volume; OTS MLFC $=$ Cambridge Neuropsychological Test Automated Battery One Touch Stocking mean latency to first choice; $\mathrm{CC}=$ corpus callosum; $\mathrm{MD}=$ mean diffusivity

3 successive boluses so that levels were elevated over a more prolonged time, increasing the likelihood that side effects would be perceived.

To date, no treatment has been demonstrated to slow symptom progression or neurodegeneration in FXTAS. In the present study, only patient 5 , the least affected patient in our cohort, experienced subjective improvement. Following the allopregnanolone treatment, he reported improved balance and restored ability to walk down stairs. He also experienced a resolution of symptoms of peripheral neuropathy, including numbness and pain. Interestingly, studies in animal neuropathic pain models have demonstrated that allopregnanolone can exert a
Fig. 5 Changes in event-related potential N400 repetition effect before and after allopregnanolone treatment. $41 \mathrm{~L}, 41 \mathrm{R}=$ approximately over Brodmann area 41; WL, WR = over Wernicke's area and right hemisphere homologue
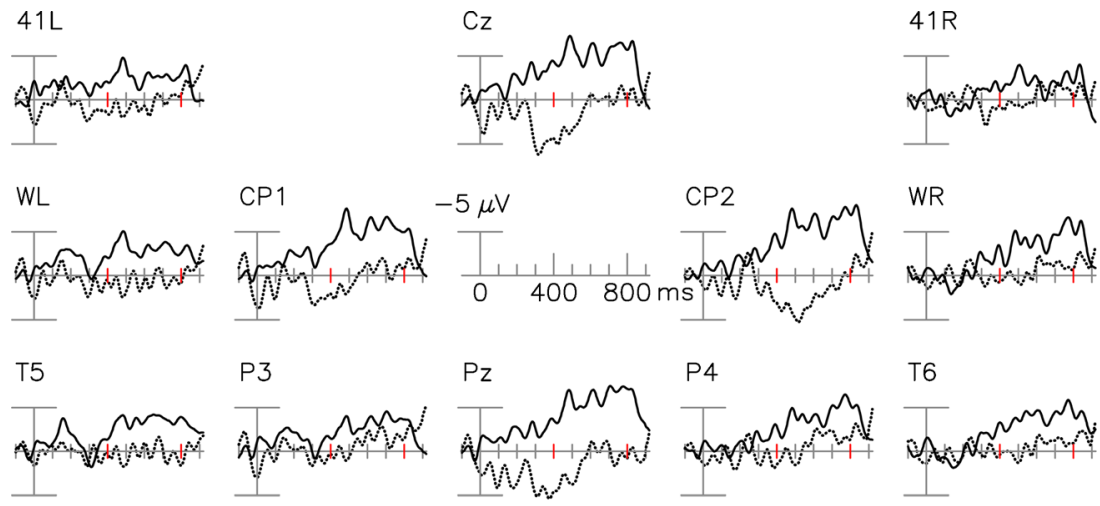

T6
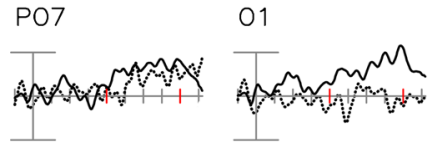

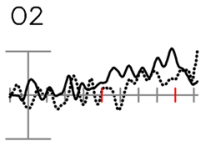

PO8

Baseline

Follow-up 
reparative effect $[48,49]$. In addition, in human studies, oral administration of synthetic allopregnanolone-like compounds exhibited antinociceptive properties. The other 5 patients did not experience improvement in their ataxia or balance symptoms, and none of the patients experienced improvement in their tremor. These subjective experiences are validated by the lack of significant improvement in the CATSYS measures of tremor or balance.

Cognitively, the patients demonstrated significant improvement in executive function and episodic memory and learning, the most affected domains in the 6 patients at baseline and in prior studies of patients with FXTAS [50]. The results support anecdotal reports from the patients and their families that cognitive processing and conversational abilities were improved following the allopregnanolone treatment. Since this was not a randomized controlled study, we cannot rule out the possibility that these improvements were due to a placebo effect, although the improvements seen in ERP results, neuropsychological testing, and MRI findings suggest that allopregnanolone could have had a disease-modifying effect.

We evaluated brain structures that are affected in FXTAS and could potentially have shown effects of allopregnanolone, including the hippocampus, the site of adult neurogenesis [51]; the amygdala, where neurosteroid exerts anxiolytic effect [52, 53]; and the corpus callosum, where the white matter structure is severely damaged in FXTAS [54-59]. Examination of serial MRI scans acquired before the treatment confirmed progressive atrophy in all 3 structures, structural deterioration in the corpus callosum, and expansion of subcortical FLAIR hyperintensities. Importantly, the directions of the pretreatment changes generally displayed consistency across the data points. Compared with the controls, the patients exhibited acceleration of hippocampal atrophy, subcortical hyperintensity expansion, and structural deterioration in the corpus callosum before the treatment. After the treatment, individual patients showed improvement in some of the MRI measurements, while showing no change or worsening in others. None of the changes in MRI measurements reached statistical significance at the 0.05 level, most likely because of significant interpatient variability. In light of variabilities in patients' baseline conditions, as well as in changes after the treatment, we performed correlational analyses to identify baseline MRI measurements that could predict functional changes. The significant correlations between baseline MRI measurements and functional improvements indicate that allopregnanolone may show effects in improving executive function in patients with relatively healthy hippocampus and corpus callosum and in alleviating psychological symptoms in patients with small hippocampus and amygdala.

Only one previous controlled trial has been performed in individuals with FXTAS, a memantine trial lasting 1 year, but this treatment did not improve tremor, ataxia, or executive function [22]. However, we found that the N400 repetition effect amplitude, thought to index semantic processing and verbal learning/memory, displayed improvements after the memantine treatment compared with placebo, and predicted verbal learning and memory performance in the FXTAS group (but not in a group with prodromal/early Alzheimer disease) $[60,61]$. The results suggest that the N400 repetition effect, primarily originating from the temporal neocortex [62, 63], is sensitive to memory changes in FXTAS. In the current open-label study, ERP assessments were conducted in 4 patients, all of whom demonstrated improvements in the N400 repetition effect.

Notably, this preliminary trial was conducted to obtain evidence of treatment benefits in a small sample, and thus the study was not powered to detect statistical significance while controlling for multiple comparisons. The substantial amount of interpatient variability is likely another contributing factor for the nonsignificant results, given the small sample size. Despite all statistical analyses provided in the study, it should be noted that all analyses were based on a small sample size as to provide preliminary evidence on the clinical efficacy of allopregnanolone; thus, an extra level of caution is warranted in using the results. The lack of a placebo group is an additional study limitation. Despite these limitations, our findings demonstrate the promise of allopregnanolone in treating FXTAS, which will be subject to future confirmatory studies with larger numbers of patients.

In summary, this open-label treatment trial has provided preliminary evidence that weekly infusion of allopregnanolone in individuals with FXTAS may improve deficits in executive function, learning, and memory. We did not, however, obtain evidence of a benefit for the hallmark neurological symptoms of tremor and ataxia. Subtle improvements on some MRI measurements were also noted for some patients, suggesting that allopregnanolone could be disease-modifying after only 12 weeks of treatment, possibly through an effect on neurogenesis or via neuroprotection. These preliminary results indicate that controlled trials, possibly over a longer treatment period, are warranted.

Acknowledgments We thank Gerhard Bauer for GMP manufacturing. This study was made possible by private donations to Fragile Xassociated tremor/ataxia research and by National Institute of Child Health and Human Development (NICHD) grant R01 HD036071 to R.J.H. Additional support was provided by the MIND Institute Intellectual and Developmental Disabilities Research Center (NICHD grant U54 HD079125) and by the Office of the Assistant Secretary of Defense for Health Affairs under Award No. W81XWH-09-1-0746 to M.A.R. Opinions, interpretations, conclusions, and recommendations are those of the authors and are not necessarily endorsed by the Department of Defense. The event-related potential work received support from National Institute on Aging grant R01 AG04825 to J.M.O.

Required Author Forms Disclosure forms provided by the authors are available with the online version of this article. 


\section{References}

1. Hagerman RJ, Leehey M, Heinrichs W, et al. Intention tremor, parkinsonism, and generalized brain atrophy in male carriers of fragile X. Neurology 2001;57(1):127-130.

2. Berry-Kravis E, Abrams L, Coffey SM, et al. Fragile X-associated tremor/ataxia syndrome: clinical features, genetics, and testing guidelines. Mov Disord 2007;22(14):2018-1030.

3. Jacquemont S, Hagerman RJ, Leehey M, et al. Fragile X premutation tremor/ataxia syndrome: molecular, clinical, and neuroimaging correlates. Am J Hum Genet 2003;72(4):869-878.

4. Tassone F, Berry-Kravis EM, editors. Fragile X-associated tremor ataxia syndrome (FXTAS). New York, NY: Springer; 2010.

5. Loesch DZ, Cook M, Litewka L, et al. A low symptomatic form of neurodegeneration in younger carriers of the FMR1 premutation, manifesting typical radiological changes. J Med Genet 2008;45(3):179-181.

6. Cohen S, Masyn K, Adams J, et al. Molecular and imaging correlates of the fragile $\mathrm{X}$-associated tremor/ataxia syndrome. Neurology 2006;67(8):1426-1431.

7. Brunberg JA, Jacquemont S, Hagerman RJ, et al. Fragile $X$ premutation carriers: characteristic MR imaging findings of adult male patients with progressive cerebellar and cognitive dysfunction. AJNR Am J Neuroradiol 2002;23(10):1757-1766.

8. Hashimoto R, Javan AK, Tassone F, Hagerman RJ, Rivera SM. A voxel-based morphometry study of grey matter loss in fragile $\mathrm{X}$ associated tremor/ataxia syndrome. Brain 2011;134(Pt 3):863-878.

9. Leow A, Harvey D, Goodrich-Hunsaker NJ, et al. Altered structural brain connectome in young adult fragile $\mathrm{X}$ premutation carriers. Hum Brain Mapp 2014;35(9):4518-4530.

10. Wang JY, Hessl D, Hagerman RJ, et al. Abnormal trajectories in cerebellum and brainstem volumes in carriers of the fragile $\mathrm{X}$ premutation. Neurobiol Aging 2017;55:11-19.

11. Hall DA, Leehey MA, Berry-Kravis E, Hagerman RJ. Treatment and management of FXTAS. In: Tassone F, Hall DA, editors. FXTAS, FXPOI, and Other Premutation Disorders. AG Switzerland: Springer International Publishing; 2016. p. 181-98.

12. Singh C, Liu L, Wang JM, et al. Allopregnanolone restores hippocampal-dependent learning and memory and neural progenitor survival in aging $3 \mathrm{xTgAD}$ and nonTg mice. Neurobiol Aging 2012;33(8):1493-1506.

13. Brinton RD. Neurosteroids as regenerative agents in the brain: therapeutic implications. Nat Rev Endocrinol 2013;9(4):241-250.

14. Djebaili M, Hoffman SW, Stein DG. Allopregnanolone and progesterone decrease cell death and cognitive deficits after a contusion of the rat pre-frontal cortex. Neuroscience 2004;123(2):349-359.

15. Djebaili M, Guo Q, Pettus EH, Hoffman SW, Stein DG. The neurosteroids progesterone and allopregnanolone reduce cell death, gliosis, and functional deficits after traumatic brain injury in rats. $\mathrm{J}$ Neurotrauma 2005;22(1):106-118.

16. Cao Z, Hulsizer S, Tassone F, et al. Clustered burst firing in FMR1 premutation hippocampal neurons: amelioration with allopregnanolone. Hum Mol Genet 2012;21(13):2923-2935.

17. Timby E, Balgard M, Nyberg S, et al. Pharmacokinetic and behavioral effects of allopregnanolone in healthy women. Psychopharmacology (Berl) 2006;186(3):414-424.

18. Broomall E, Natale JE, Grimason M, et al. Pediatric superrefractory status epilepticus treated with allopregnanolone. Ann Neurol 2014;76(6):911-915.

19. Vaitkevicius H, Bobb W, Rosenthal ES, et al. First-in-man allopregnanolone use in super-refractory status epilepticus. Ann Clin Transl Neurol 2017;4:411-414.

20. Filipovic-Sadic S, Sah S, Chen L, et al. A novel FMR1 PCR method for the routine detection of low abundance expanded alleles and full mutations in fragile X syndrome. Clin Chem 2010;56(3):399-408.
21. Giulivi C, Zhang YF, Omanska-Klusek A, et al. Mitochondrial dysfunction in autism. JAMA 2010;304(21):2389-2396.

22. Seritan AL, Nguyen DV, Mu Y, et al. Memantine for fragile Xassociated tremor/ataxia syndrome: a randomized, double-blind, placebo-controlled trial. J Clin Psychiatry 2014;75:264-271.

23. Delis DC, Kramer JH, Kaplan E, Ober B. California verbal learning test-second edition. San Antonio, TX: Psychological Corporation; 2000.

24. Wechsler D. Wechsler Memory Scale-Fourth Edition. San Antonio, TX: Pearson; 2009.

25. Folstein MF, Folstein SE, McHugh PR. "Mini-mental state". A practical method for grading the cognitive state of patients for the clinician. J Psychiatr Res 1975;12(3):189-198.

26. Grigsby J. The Behavioral Dyscontrol Scale: Manual (2nd Ed.). Denver, CO: Authors; 1996.

27. Kaye K, Grigsby J, Robbins LJ, Korzun B. Prediction of independent functioning and behavior problems in geriatric patients. J Am Geriatr Soc 1990;38(12):1304-1310.

28. Fray PJ, Robbins TW. CANTAB battery: proposed utility in neurotoxicology. Neurotoxicol Teratol 1996;18(4):499-504.

29. Spreen O, Strauss E. A compendium of neuropsychological tests: administration, norms and commentary. New York: Oxford University Press; 1998.

30. Derogatis LR. Symptom Checklist-90-R: administration, scoring and procedures manual. 3rd ed. Minneapolis, MN: National Computer Systems, Inc.; 1994.

31. Beck AT, Epstein N, Brown G, Steer RA. An inventory for measuring clinical anxiety: psychometric properties. J Consult Clin Psychol 1988;56(6):893-897.

32. Aguilar D, Sigford KE, Soontarapornchai K, et al. A quantitative assessment of tremor and ataxia in FMR1 premutation carriers using CATSYS. Am J Med Genet A 2008;146A:629-635.

33. Narcisa V, Aguilar D, Nguyen DV, et al. A quantitative assessment of tremor and ataxia in female FMR1 premutation carriers using CATSYS. Curr Gerontol Geriatr Res 2011;2011:484713.

34. Ardekani BA, Bachman AH. Model-based automatic detection of the anterior and posterior commissures on MRI scans. Neuroimage 2009;46(3):677-682.

35. Mori S, Crain BJ, Chacko VP, van Zijl PC. Three-dimensional tracking of axonal projections in the brain by magnetic resonance imaging. Ann Neurol 1999;45(2):265-269.

36. Tustison NJ, Avants BB, Cook PA, et al. N4ITK: improved N3 bias correction. IEEE Trans Med Imaging 2010;29(6):1310-1320.

37. Smith SM, Jenkinson M, Woolrich MW, et al. Advances in functional and structural MR image analysis and implementation as FSL. Neuroimage 2004;23(Suppl. 1):S208-S219.

38. Wang H, Pouch A, Takabe M, et al. Multi-atlas segmentation with robust label transfer and label fusion. Inform Process Med Imaging 2013;23:548-559.

39. Wang H, Das SR, Suh JW, et al. A learning-based wrapper method to correct systematic errors in automatic image segmentation: consistently improved performance in hippocampus, cortex and brain segmentation. Neuroimage 2011;55(3):968-985.

40. Wang JY, Ngo MM, Hessl D, Hagerman RJ, Rivera SM. Robust Machine learning-based correction on automatic segmentation of the cerebellum and brainstem. PLOS ONE 2016;11(5):e0156123.

41. Wang JY, Hagerman RJ, Rivera SM. A multimodal imaging analysis of subcortical gray matter in fragile $\mathrm{X}$ premutation carriers. Mov Disord 2013;28(9):1278-1284.

42. Greve DN, Fischl B. Accurate and robust brain image alignment using boundary-based registration. Neuroimage 2009;48(1):63-72.

43. Schmidt P, Gaser C, Arsic M, et al. An automated tool for detection of FLAIR-hyperintense white-matter lesions in multiple sclerosis. Neuroimage 2012;59(4):3774-3783.

44. Griffanti L, Zamboni G, Khan A, et al. BIANCA (Brain Intensity AbNormality Classification Algorithm): a new tool for automated 
segmentation of white matter hyperintensities. Neuroimage 2016;141:191-205.

45. Olichney JM, Chan S, Wong LM, et al. Abnormal N400 word repetition effects in fragile $\mathrm{X}$-associated tremor/ataxia syndrome. Brain 2010;133(Pt 5):1438-1450.

46. Team RDC. R: a language and environment for statistical computing. Vienna, Austria: R Foundation for Statistical Computing; 2014.

47. Winter B. Linear models and linear mixed effects models in $\mathrm{R}$ with linguistic applications. Available at: http://arxiv.org/pdf/1308.5499. pdf. Accessed June 14, 2017.

48. Meyer L, Patte-Mensah C, Taleb O, Mensah-Nyagan AG. Allopregnanolone prevents and suppresses oxaliplatin-evoked painful neuropathy: multi-parametric assessment and direct evidence. Pain 2011;152(1):170-181.

49. Patte-Mensah C, Meyer L, Taleb O, Mensah-Nyagan AG. Potential role of allopregnanolone for a safe and effective therapy of neuropathic pain. Prog Neurobiol 2014;113:70-78.

50. Grigsby J, Cornish K, Hocking D, et al. The cognitive neuropsychological phenotype of carriers of the FMR1 premutation. J Neurodevelop Disord 2014;6(1):28.

51. Lieberwirth C, Pan Y, Liu Y, Zhang Z, Wang Z. Hippocampal adult neurogenesis: Its regulation and potential role in spatial learning and memory. Brain Res 2016;1644:127-140.

52. Akwa Y, Purdy RH, Koob GF, Britton KT. The amygdala mediates the anxiolytic-like effect of the neurosteroid allopregnanolone in rat. Behav Brain Res 1999;106(1-2):119-125.

53. Wang C, Marx CE, Morrow AL, Wilson WA, Moore SD. Neurosteroid modulation of GABAergic neurotransmission in the central amygdala: a role for NMDA receptors. Neurosci Lett 2007;415(2):118-123.

54. Adams PE, Adams JS, Nguyen DV, et al. Psychological symptoms correlate with reduced hippocampal volume in fragile $\mathrm{X}$ premutation carriers. Am J Med Genet B Neuropsychiatr Genet 2009;153B(3):775-785.

55. Hessl D, Rivera S, Koldewyn K, et al. Amygdala dysfunction in men with the fragile X premutation. Brain 2007;130(Pt 2):404-416.

56. Koldewyn K, Hessl D, Adams J, et al. Reduced hippocampal activation during recall is associated with elevated FMR1 mRNA and psychiatric symptoms in men with the Fragile X premutation. Brain Imaging Behav 2008;2(2):105-116.

57. Wang JY, Hessl DH, Hagerman RJ, Tassone F, Rivera SM. Agedependent structural connectivity effects in fragile $\mathrm{x}$ premutation. Arch Neurol 2012;69(4):482-489.

58. Wang JY, Hessl D, Schneider A, Tassone F, Hagerman RJ, Rivera SM. Fragile X-associated tremor/ataxia syndrome: influence of the FMR1 gene on motor fiber tracts in males with normal and premutation alleles. JAMA Neurol 2013;70(8):1022-1029.

59. Greco CM, Berman RF, Martin RM, et al. Neuropathology of fragile X-associated tremor/ataxia syndrome (FXTAS). Brain 2006;129(Pt 1):243-255.

60. Yang JC, Chi L, Teichholtz S, et al. ERP abnormalities elicited by word repetition in fragile X-associated tremor/ataxia syndrome (FXTAS) and amnestic MCI. Neuropsychologia 2014;63:34-42.

61. Yang JC, Niu YQ, Simon C, et al. Memantine effects on verbal memory in fragile X-associated tremor/ataxia syndrome (FXTAS): a doubleblind brain potential study. Neuropsychopharmacology 2014;39(12): 2760-2768.

62. Halgren E, Dhond RP, Christensen N, et al. N400-like magnetoencephalography responses modulated by semantic context, word frequency, and lexical class in sentences. Neuroimage 2002;17(3): 1101-1116.

63. Nobre AC, Allison T, McCarthy G. Word recognition in the human inferior temporal lobe. Nature 1994;372(6503):260-263. 